\title{
Dynamics of a Versatile Sliding Control
}

\author{
LIANG-WEY CHANG* \\ Center for Biomedical Engineering, College of Medicine, National Taiwan University, Taipei, Taiwan, R.O.C.
}

Editor: E. P. Ryan

Received December 9, 1991; Revised November 13, 1992

\begin{abstract}
The dynamics of a versatile sliding control is presented, which utilizes a low-pass filter for second-order $s$ dynamics and a high-pass (or band-pass) filter for an error dynamics in series. The versatile sliding control places zeros within the error dynamics and applies a second-order sliding condition. The second-order $s$ dynamics sharply rejects unwanted high-frequency signals that include unmodeled dynamics and disturbances. The bandwidth of the $s$ dynamics is designed low such that the excitation to the error dynamics will be minimum. With a lower bound of damping ratio for the $s$ dynamics, the thickness of the boundary layer can be self-tuned by the placed zeros and the spring constant within the sliding condition to fine tune the $s$ dynamics and the error dynamics. The trade-off between tracking accuracy and the robustness to the uncertainties no longer exists. The control exhibits greater flexibility in design and better performance than its counterpart with a first-order sliding condition.
\end{abstract}

\section{Introduction}

The sliding (mode) control method utilizes the Variable Structure Concept [6], [11] and provides a simple and systematic way to intentionally alter control structure such that the control system is insensitive to the modeling error and disturbances. The control drives the state onto a user-designed surface (the sliding surface) and maintains the state on the surface until a desired state is reached. The Lyapunov stability criterion is used to generate a sliding condition such that the state can reach the sliding surface within a finite time and remain on the surface.

The sliding control has been reviewed by Utkin [10] and DeCarlo et al. [3] where the sliding surface was defined in terms of state variables. With the state-variable expression, the system dynamics on the sliding surface was derived by the equivalent control method and the sliding surface was designed based on the requirement of having a stable system dynamics [4]. Recently, the sliding surface was defined in terms of tracking errors [5], [7] with which a sliding mode can be readily obtained as long as a stable linear differential operator on the tracking errors is selected. The tracking-error approach results in a direct and simpler design of sliding surfaces.

The switched control was first developed utilizing the sliding-control concept. A buffer element, i.e., the $s$ dynamics, which describes dynamics of the sliding surface variable $s$, plays a central role to filter the uncertainty and provide system robustness. The resulting high-frequency output from the switched $s$ dynamics is filtered by a low-pass filter of error dynamics and perfect regulation is obtained. The high-frequency switching results

\footnotetext{
The author was previously with the Department of Mechanical Engineering at the U.S. Naval Postgraduate School, Monterey, California.
} 
in chattering phenomenon of the control input that is not desirable for mechanical devices because of noise generation and wear.

Continuous (smoothing) control has been introduced to eliminate high-frequency switching at the sacrifice of perfect regulation. A continuous tracking control for nonlinear time-varying dynamical systems [7] was developed, where the sliding surface was defined in tracking errors and directly linked to error dynamics. The high-frequency switched $s$ dynamics was transformed into a first-order low-pass filter inside the boundary layer. However, the control scheme resulted in a trade-off between robustness and tracking accuracy. With additional integral control, continuous control eliminates steady-state error. As the integral control action destabilizes the control system, the system response tends to be oscillatory. The capability of the sliding control in shaping the system dynamics is too limited to meet the no-overshoot (no-oscillation) requirement and the system response in turn is sluggish (longer rise time and settling time).

The trade-off between robustness and tracking accuracy was further refined [8], and the control scheme has been applied to various dynamical systems [9], [12]. Ambrosino (1984) also attempted to eliminate partially the chattering so that the tracking error would not deteriorate considerably.

To eliminate the trade-off, the second-order sliding condition [2] was also explored to develop a simple robust sliding tracking control for time-varying nonlinear systems. The control, which places zeros in the error dynamics and applies a second-order sliding condition, provides better tuning capability than its counterpart with the first-order sliding condition. The relative stability is tightly controlled and the tracking errors are improved. The sliding control utilizes a low-pass filter and a high-pass (or band-pass) filter in series. The low-pass filter rejects the unmodeled dynamics and environmental disturbances, and the high-pass filter practically keeps the desired system response intact from the low-frequency excitation generated from the low-pass filter.

In this paper, the sliding control with second-order sliding condition (Versatile Sliding Control) will be further investigated particularly regarding dynamic behavior and the tuning efficiency. For simplicity, a SISO (Single-Input-Single-Output) system will be used to demonstrate concepts. Finally, an example of a single-arm manipulator with varying payloads will be given to show the design, performance and robustness of the control system.

\section{System Model and Uncertainty}

The sliding control is a deterministic process for dynamical systems and deals with modeling errors as well as environmental disturbances. A well-defined bounding of the uncertainty along with a deterministic physical model is therefore required.

Consider a scalar $n$ th-order time-varying dynamical system

$$
x^{(n)}=b u+f+d
$$

where $x^{(n)}$ is the $n$ th-order time-derivative of an output variable of interest $x . u$ is a control input and $d$ is a time-varying disturbance. $b$ and $f$ are the functions of output variables and time. Equation (2.1) represents a canonical I/O form of physical models of nonlinear 
time-varying dynamical systems. A nominal mathematical model of the system can be obtained and written as

$$
x^{(n)}=\hat{b} u+\hat{f}
$$

$\hat{b}$ and $\hat{f}$ are the nominal values of $b$ and $f$ and can be obtained through experiment or theoretical evaluations.

The bounding of the uncertainty is based on an assumption that the modeling error is bounded. The manner of bounding could have many forms. A type of confinement being adopted in this paper is [8]

$$
|f-\hat{f}|<F \quad \text { and } \quad \beta^{-1} \leq \frac{\hat{b}}{b} \leq \beta
$$

where $F=\left(f_{\max }-f_{\min }\right) / 2$ and $\beta=\left(b_{\max } / b_{\min }\right)^{1 / 2}$. It implies that the system parameters and their nominal values are related as $\hat{f}=\left(f_{\max }+f_{\min }\right) / 2$ and $\hat{b}=\left(b_{\max } b_{\min }\right)^{\frac{1}{2}}$ although the system parameters $b, f$ and $d$ are unknown. In addition, it is assumed that the disturbance $d$ is also bounded, i.e., $|d|<D$.

\section{Sliding Surface and Second-Order Sliding Condition}

The sliding surface defines a domain to which the closed-loop control system will be driven and where the system will be maintained while the tracking error is sliding towards zero. Therefore, the sliding control initially attracts the system towards the surface and the system is required to be asymptotically stable while on the sliding surface. There is a need to introduce a variable $s$ which connects the closed-loop dynamic behavior and the sliding surface. A sliding condition is needed by which the control will be designed to drive the variable towards zero, i.e., sliding surface, in an asymptotically stable manner. When the sliding surface has been reached and the state remains on the surface, the value of the variable stays at zero and the system is in the sliding mode. The sliding surface, $s=0$, must be designed such that the dynamics of the tracking error is also asymptotically stable in the sliding mode.

The second-order sliding condition derived from the Lyapunov stability criterion specifies the dynamic behavior of the variable $s$. The purpose of using the second-order sliding condition is twofold: (1) to increase the order of the $s$ dynamics and increase the activity of the $s$ dynamics and (2) to provide more tuning parameters and increase tuning capability. The application of the second-order sliding control results in a control design with better system robustness and higher tracking accuracy. Let a Lyapunov function be

$$
V=\frac{1}{2} \dot{s} \cdot \dot{s}+\frac{1}{2} s \cdot \omega_{n}^{2} \cdot s
$$

To have stable $s$ dynamics, $\dot{V} \leq 0$, i.e.,

$$
\dot{s}\left(\ddot{s}+\omega_{n}^{2} s\right) \leq 0
$$


Equation (3.2) represents a stability criterion for a mass-spring-damper system, i.e., a massspring system with a positive damping. The spring constant $\omega_{n}^{2}$ will reflect the stiffness of the $s$ dynamics and can be adjusted to allow for more tuning flexibility. Due to the fact that the $s$ motion may stop and stay at a nonzero position when $\dot{V}=0$, Equation (3.2) is rewritten to be

$$
\dot{s}\left(\ddot{s}+\omega_{n}^{2} s\right) \leq-\eta|\dot{s}|
$$

The above equation also allows one to adjust the approaching speed towards the sliding surface by varying $\eta$.

In the sliding mode where $s$ and its derivatives are zero, the closed-loop system dynamics represented by its tracking-error dynamics must be asymptotically stable. Let $e=x-x_{d}$ be the tracking error, i.e., the difference between the output measurement and the desired output. The error dynamics is defined to be

$$
\left(\frac{d}{d t}+z_{0}\right) s=\prod_{i=1}^{n}\left(\frac{d}{d t}+p_{i}\right) \int_{0}^{t} e d t
$$

where the $p$ 's and $z_{0}$ are assigned to locate the poles and zero for the error dynamics. The polynomial version of the above equation is

$$
\dot{s}+z_{0} s=e^{(n-1)}+c_{n-1} e^{(n-2)}+c_{n-2} e^{(n-3)}+\cdots+c_{0} \int_{0}^{t} e d t
$$

The above equation indicates that the $s$ terms in the left-hand side are inputs to the error dynamics, and the error $e$ becomes a filtered version of $s$. Since the physical relationship between $s$ and tracking error is also governed by the equation, the closed-loop control system is asymptotically stable given that the $s$ dynamics is asymptotically stable. The above formula could have been more sophisticated if more integral terms of $s$ and $e$ had been included. For example, the error dynamics could have been defined as

$$
\begin{aligned}
\dot{s}+ & z_{0} s+z_{1} \int_{0}^{t} s d t+\cdots+z_{p} \int_{0}^{t} \ldots \int_{0}^{t} s(d t)^{p} \\
= & e^{(n-1)}+c_{n-1} e^{(n-2)}+c_{n-2} e^{(n-3)}+\cdots+c_{0} \int_{0}^{t} e d t \\
& +\cdots+c_{1-m} \int_{0}^{t} \cdots \int_{0}^{t} e(d t)^{m}
\end{aligned}
$$

where $p$ and $m$ are integers. For simplicity, only Equation (3.5) will be adopted in this paper. It will be shown that $s$ is influenced by the control input $u$ and therefore the $s$ dynamics can be modulated by the control input. By differentiating Equation (3.5),

$$
\ddot{s}+z_{0} \dot{s}=e^{(n)}+c_{n-1} e^{(n-1)}+c_{n-2} e^{(n-2)}+\cdots+c_{1} \dot{e}+c_{0} e
$$


The control input $u$ can influence the $s$ dynamics through the following equation:

$$
\ddot{s}+z_{0} \dot{s}=b u+f+d-x_{d}^{(n)}+\varepsilon
$$

where

$$
\varepsilon=c_{n-1} e^{(n-1)}+c_{n-2} e^{(n-2)}+\cdots+c_{1} \dot{e}+c_{0} e
$$

The role of the control $u$ is to shape the $s$ dynamics such that the sliding surface can be reached in a finite time. Once the system is in the sliding mode, the asymptotically stable error dynamics (Equation (3.5)) guarantees zero steady-state tracking error.

\section{A Versatile Sliding Control Algorithm}

The sliding control law requires two combined actions: (1) an equivalent control that keeps the error dynamics asymptotically stable in the sliding mode, and (2) an additional switched control that maintains the state on the sliding surface. The switched control is switching about $\dot{s}=0$ based on the sign of the indicator $\dot{s}$ which determines the Lyapunov stability according to the second-order sliding condition since it determines whether the damping is positive or not. The switched control is the key to the system robustness and usually results in a fast motion to bring the state onto the sliding surface where a slower motion proceeds until a desired state is reached.

To eliminate the chattering, the saturation function is utilized along with adopting the boundary-layer concept. The continuous control law is designed as

$$
u=\hat{b}^{-1}\left(\hat{u}-k s a t\left(\frac{\dot{s}}{\phi}\right)\right)
$$

where

$$
\hat{u}=-\hat{f}+x_{d}^{(n)}-\varepsilon+z_{0} \dot{s}-\omega_{n}^{2} \int_{0}^{t} \dot{s} d t
$$

and

$$
\dot{s}=e^{(n-1)}+c_{n-1} e^{(n-2)}+c_{n-2} e^{(n-3)}+\cdots+c_{0} \int_{0}^{t} e d t-z_{0} \int_{0}^{t} \dot{s} d t
$$

where $\phi$ is the thickness of the boundary layer for the parameter $\dot{s}$. Outside the boundary layer, $|\dot{s}| \geq \phi$, the second-order sliding condition is applied to govern the stability of the discontinuous $s$ dynamics. Inside the boundary layer, $|\dot{s}|<\phi$, the continuous control law gives smooth $s$ dynamics.

The constant gain $k$ is selected to satisfy the sliding condition in order to guarantee the asymptotic stability of the $s$ dynamics outside the boundary layer. By adding $\omega_{n}^{2} s$ to the Equation (3.8), the second-order sliding condition (Equation (3.3)) is given as

$$
\dot{s} \cdot\left(b u+f+d-x_{d}^{(n)}+\varepsilon-z_{0} \dot{s}+\omega_{n}^{2} s\right) \leq-\eta|\dot{s}|
$$




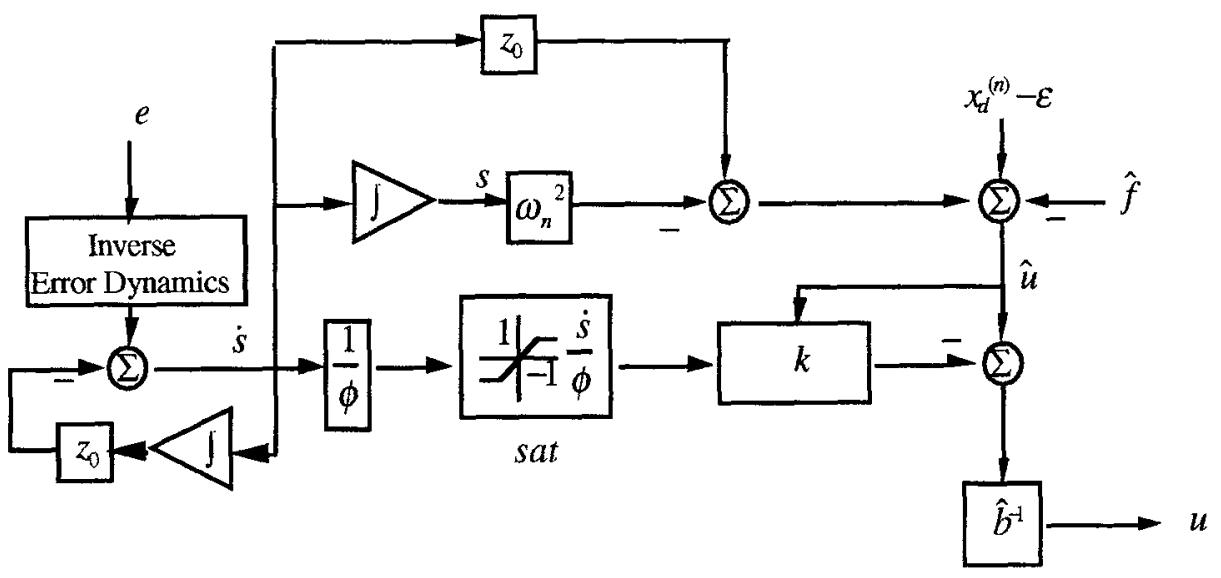

Figure 1. The versatile sliding controller.

The control input has to be designed in such a fashion that the $s$ dynamics obey the above condition. Outside the boundary layer, Equation (4.1) is equivalent to

$$
u=\hat{b}^{-1}(\hat{u}-k \operatorname{sign}(\dot{s}))
$$

By substituting Equation (4.3) into Equation (4.2) with the definition of $\hat{u}, k$ is found to be

$$
k \geq \beta(F+D+\eta)+(\beta-1)|\hat{u}|
$$

It implies that the gain $k$ is a measure of the uncertainties. For implementation, the minimum value of $k$ will be chosen to minimize the control effort. Figure 1 shows the structure of the controller which includes a block of inverse error dynamics that utilizes the system error $e$ to compute the $s$ value. Note that the inverse error dynamics is simply an inverse operation of the error dynamics (Equation (3.4)). The diagram also includes a Lyapunov control utilizing the Lyapunov stability criterion to obtain stable $s$ dynamics such that $s=0$, a sliding surface, may be reached. A saturation control eliminates the chattering and the remaining steady-state system error $e$ can also be eliminated with an integral feature in the error dynamics. The control system bandwidth can be tuned by the adjustable parameters within the system dynamics.

\section{Stability and Tuning Mechanisms}

The stability of the $s$ dynamics is to be examined since it determines the stability of the closed-loop control system. Outside the boundary layer, the stability is governed by the sliding condition by which the gain $k$ was selected. In other words, the gain is selected such that the $s$ dynamics outside the boundary layer is stable. Inside the boundary layer, 
the stability is examined from the equation which describes the smooth $s$ dynamics, i.e.,

$$
\ddot{s}+\frac{b \hat{b}^{-1} k}{\phi} \dot{s}+\omega_{n}^{2} s=(f-\hat{f})+d-\left(1-b \hat{b}^{-1}\right) \hat{u}
$$

Since $\hat{u}=-\hat{f}+x_{d}^{(n)}-\varepsilon+z_{0} \dot{s}-\omega_{n}^{2} \int_{0}^{t} \dot{s} d t$, the $s$ dynamics can be re-organized to be

$$
\begin{aligned}
\ddot{s}+ & {\left[\left(1-b \hat{b}^{-1}\right) z_{0}+b \hat{b}^{-1} \frac{k}{\phi}\right] \dot{s}+b \hat{b}^{-1} \omega_{n}^{2} s } \\
& =(f-\hat{f})+d-\left(1-b \hat{b}^{-1}\right)\left(-\hat{f}+x_{d}^{(n)}-\varepsilon\right)
\end{aligned}
$$

There are three parameters in Equation (5.2) to be determined, i.e., $z_{0}, \phi$, and $\omega_{n}$.

Note that the first-order sliding condition results in a first-order filter which only has one parameter $\phi$ to be determined and creates a trade-off between tracking accuracy and robustness. A small $\phi$ could improve the tracking accuracy while the system robustness suffers from the large bandwidth due to the small $\phi$ since the large bandwidth allows high-frequency unmodeled dynamics and disturbances to enter into the system.

The selection of the three parameters requires stability analysis of the $s$ dynamics. The values of these three parameters affect the relative stability of the $s$ dynamics which is governed by the natural frequency (bandwidth) and the damping ratio. Therefore, the tuning mechanism of the parameters will be designed through controlling the bandwidth and damping ratio. Derived from Equation (2.3), the following inequalities are useful in designing the tuning mechanism.

$$
\frac{1}{\beta} \omega_{n}^{2} \leq b \hat{b}^{-1} \omega_{n}^{2} \leq \beta \omega_{n}^{2}
$$

and

$$
(1-\beta) z_{0}+\frac{1}{\beta} \frac{k}{\phi} \leq\left(1-b \hat{b}^{-1}\right) z_{0}+b \hat{b}^{-1} \frac{k}{\phi} \leq\left(1-\frac{1}{\beta}\right) z_{0}+\beta \frac{k}{\phi}
$$

The above equations are used to determined the bounds on the parameters of $s$ dynamics, i.e., the bandwith $\omega_{s}$, and the damping ratio $\zeta_{s}$. Given the spring constant $\omega_{n}^{2}$ and the boundary layer thickness $\phi$, the lower bounds and the upper bounds of $\omega_{s}$ and $\zeta_{s}$ can be obtained as follows,

$$
\omega_{l} \leq \omega_{s} \leq \omega_{u}
$$

and

$$
\zeta_{l} \leq \zeta_{s} \leq \zeta_{u}
$$

where

$$
\omega_{l}=\frac{1}{\sqrt{\beta}} \omega_{n}
$$




$$
\begin{aligned}
& \omega_{u}=\sqrt{\beta} \omega_{n} \\
& \zeta_{l}=\frac{(1-\beta) z_{0}+\frac{1}{\beta} \frac{k}{\phi}}{2 \omega_{l}}
\end{aligned}
$$

and

$$
\zeta_{u}=\frac{\left(1-\frac{1}{\beta}\right) z_{0}+\beta \frac{k}{\phi}}{2 \omega_{u}}
$$

A time-varying $\phi$ can be derived from Equations (5.9) or (5.10) to control the $s$ dynamics and to improve the tracking performance. A non-oscillatory $s$ response is desirable and $\zeta_{1}>1$ guarantees under-damped $s$ dynamics. From Equation (5.9), by assigning $\zeta_{1}$, the boundary layer thickness can be computed as

$$
\phi=\frac{\frac{k}{\beta}}{2 \zeta_{l} \omega_{l}-(1-\beta) z_{0}}
$$

and, with the computed $\phi$, the corresponding lower (or upper) bound of the damping ratio can be obtained from Equation (5.9) (or Equation (5.10)). The range of the bandwidth for the $s$ dynamics is given by Equations $(5.5,5.7-8)$, where $\omega_{n}$ is the only control parameter for the bandwidth. To provide more tuning capability, two other control parameters, both the lower bound of the damping ratio and the specified zero, would affect $\phi$, and would further shape the $s$ dynamics and control the tracking accuracy. With this great versatility, the trade-off between tracking accuracy and robustness to the system disturbances no longer exists.

From Equation (5.11), the greater the level of uncertainties $k$, the greater is $\phi$ and this allows for more active $s$ dynamics. From Equation (5.9), with other parameters fixed, the greater the $\phi$ is, the less the damping (or more kinetic energy) is allowed for the smooth $s$ dynamics. Therefore, with greater $\phi$, the $s$ dynamics is more active and is more easily excited. In other words, the $s$ dynamics is more sensitive to its excitation with a greater value of $\phi$. The value of $\phi$ plays a critical role to fine tune the system response and requires particular attention.

Figure 2 presents a detailed $s$ dynamics from a synthesis viewpoint of the versatile sliding control system. The control is viewed as a two-stage filtering device which utilizes a lowpass filter for second-order $s$ dynamics and a high-pass (or band-pass) filter for an error dynamics in series. A desired signal (goal) can be obtained if unwanted signals are filtered by the two-stage band-pass filter. At the first stage, $s$ dynamics filters unwanted highfrequency signals that include unmodeled dynamics and disturbances. The $s$ dynamics, a low-pass filter, plays a very important role as a buffer to protect the system from being disturbed by uncertainty. The second stage of the sliding control is a high-pass (or bandpass) filter to obtain error response. The bandwidth of the $s$ dynamics will be designed low such that the high-frequency uncertainty is filtered and the excitation to the error dynamics will be minimum. 


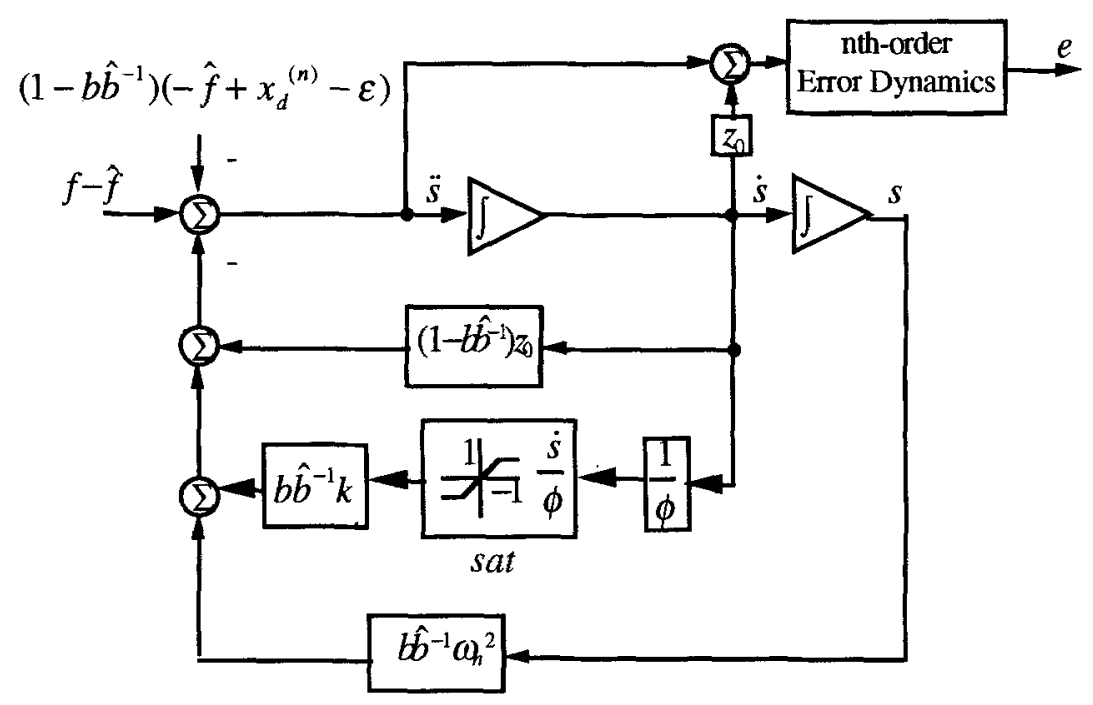

Figure 2. The structure of the versatile sliding control system.

\section{Example Presentation}

A rigid single-link robot arm equipped with robust control is used as an example to illustrate the concept and to present numerical results. The robust control is designed to control the motion of the arm for various payloads. For this case, the motor position $\theta$ on the joint is the output variable of interest. The link of the arm has a length of $L$ and a mass of $M$, and the cross sections of the link are uniform. The arm lifts a payload mass of $m_{p}$ where the maximum value is $M$. Thus the payload mass $m_{p}$ will range from 0 to $M$. The deterministic model of the plant is derived as

$$
\left(\frac{1}{3} M+m_{p}\right) L^{2} \ddot{\theta}+\left(\frac{1}{2} M+m_{p}\right) L g \cos \theta=T
$$

where $g$ is the gravitational acceleration and $T$ is the motor torque. By replacing $\theta$ by $x$ and $T$ by $u$, the canonical form of the model is written as

$$
\ddot{x}=b u+f+d
$$

where

$$
\begin{aligned}
& b=\frac{1}{\left(\frac{1}{3} M+m_{p}\right) L^{2}} \\
& d=0 \quad \text { and } \quad f=-\frac{\left(\frac{1}{2} M+m_{p}\right) g \cos \theta}{\left(\frac{1}{3} M+m_{p}\right) L}
\end{aligned}
$$


Since $0 \leq m_{p} \leq M$, for $0 \leq \theta \leq 90^{\circ}, b_{\min }, b_{\max }, f_{\min }$ and $f_{\max }$ are listed as,

$$
\begin{aligned}
& b_{\min }=\frac{3}{4 M L^{2}}, \quad b_{\max }=\frac{3}{M L^{2}} \\
& f_{\min }=-\frac{3 g}{2 L}, \text { and } f_{\max }=0
\end{aligned}
$$

Now, a nominal model with its uncertainty confinement is given as

$$
\begin{aligned}
& \ddot{x}=\hat{b} u+\hat{f} \\
& |f-\hat{f}|<F \text { and } \beta^{-1} \leq \frac{\hat{b}}{b} \leq \beta
\end{aligned}
$$

where

$$
\begin{aligned}
& \hat{b}=\frac{3}{2 M L^{2}}, \hat{f}=-\frac{3 g}{4 L}, \\
& F=\frac{3 g}{4 L} \quad \text { and } \quad \beta=2
\end{aligned}
$$

For the numerical results, the plant has the following data: $M=4(\mathrm{Kg}), L=1(\mathrm{~m})$, and $m_{p}=4(\mathrm{Kg})$. Note that the payload mass of the plant has been chosen as the maximum value for which the robust control is designed. This value was selected in order to run a severe test on the robust control. The desired trajectory was selected to be

$$
x_{d}= \begin{cases}t & \text { for } 0 \leq t<1 \\ 1 & \text { for } 1 \leq t<2 \\ 3-t & \text { for } 2 \leq t<3 \\ 0 & \text { for } t \leq 3\end{cases}
$$

where the unit of time $t$ is a second. The system was simulated with zero initial conditions. The time interval for numerical integration was $0.01(\mathrm{sec})$ unless different values are stated. To gain enough speed and critically damped response, the error dynamics was set for a bandwidth of $10(\mathrm{rad} / \mathrm{sec})$, i.e., first pole $-p_{1}=-10$ and second pole $-p_{2}=-10$. The parameter for controlling the approaching speed outside the boundary layer, $\eta$, was assigned to be 10 . Any chattering of the manipulator will be eliminated by utilizing continuous sliding control. The spring constant of the second order sliding condition was selected as $\omega_{n}^{2}=1$ to keep the bandwidth of the $s$ dynamics low. Along with the selection of $\zeta_{l}$ (the lower bound of the damping ratio) for the $s$ dynamics, the zero $z_{0}$ should be placed such that the $s$ dynamics can be fine tuned. Note that the value of $\zeta_{l}$ and $z_{0}$ should be chosen such that $\phi \geq 0$. The value of $z_{0}$ affects the filtering level of error dynamics and the thickness of the boundary layer (Equation (5.11)). With a fixed $\zeta_{1}$, the smaller the value of $z_{0}$, the more 


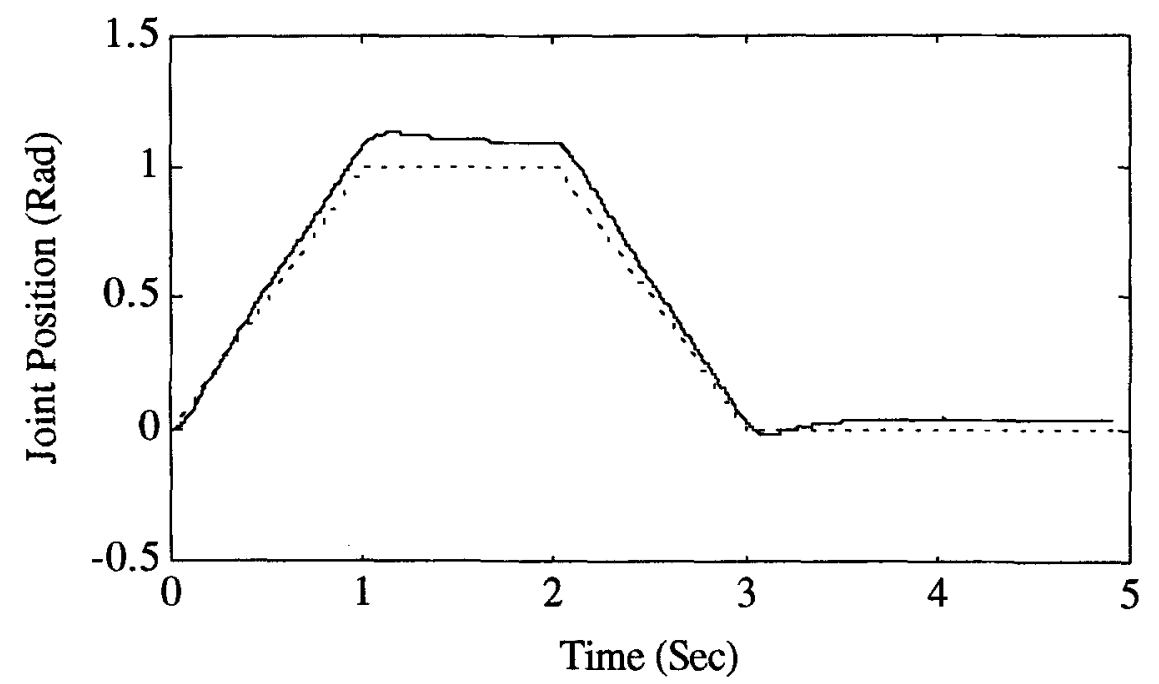

Figure 3. The tracking performance of a versatile sliding control $\left(\zeta=1\right.$ and $z_{0}=100$ ) (a dotted line for desired trajectory).

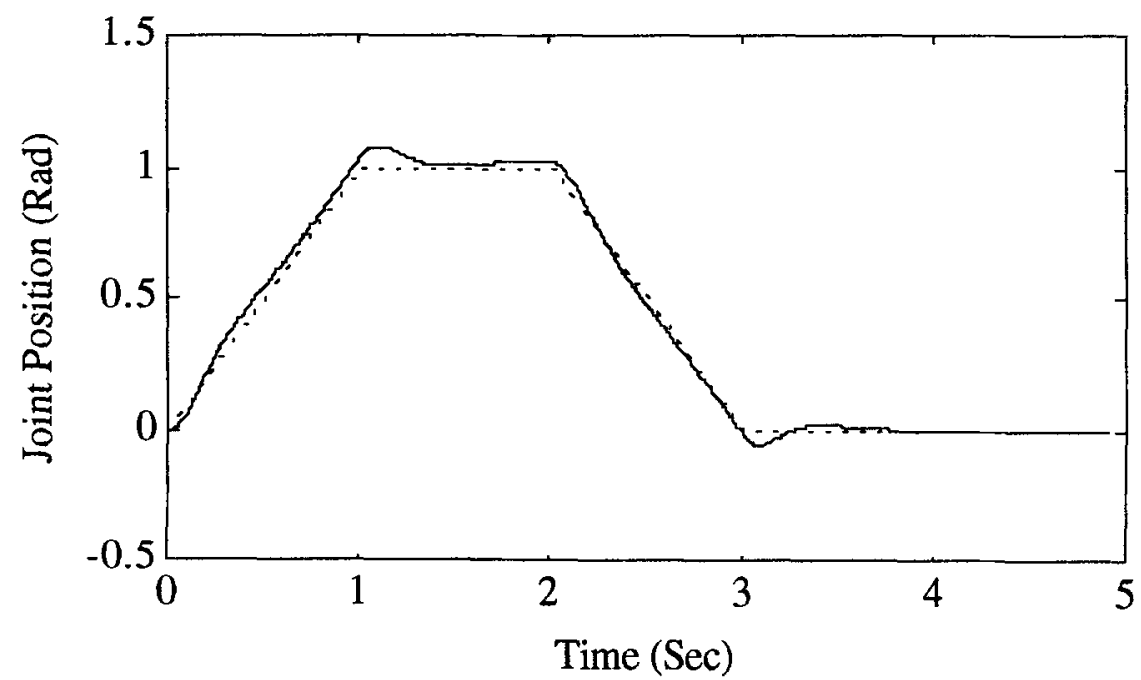

Figure 4. The tracking performance of a versatile sliding control $\left(\zeta_{l}=1\right.$ and $z_{0}=1$ ) (a dotted line for desired trajectory).

active the $s$ dynamics, but the better is filtering for the low frequencies. Given $\zeta_{l}=1$, Figures 3-5 present the tracking performance due to three values of $z_{0}$, i.e., 100,1 , and -1 . By comparing Figures 3 and 4 , it is observed that decreasing the value of the specified zero $-z_{0}$ speeds up the response since the low frequencies (slow motion components) of the $s$ dynamics output are filtered by the error dynamics. However, on the other hand, with 


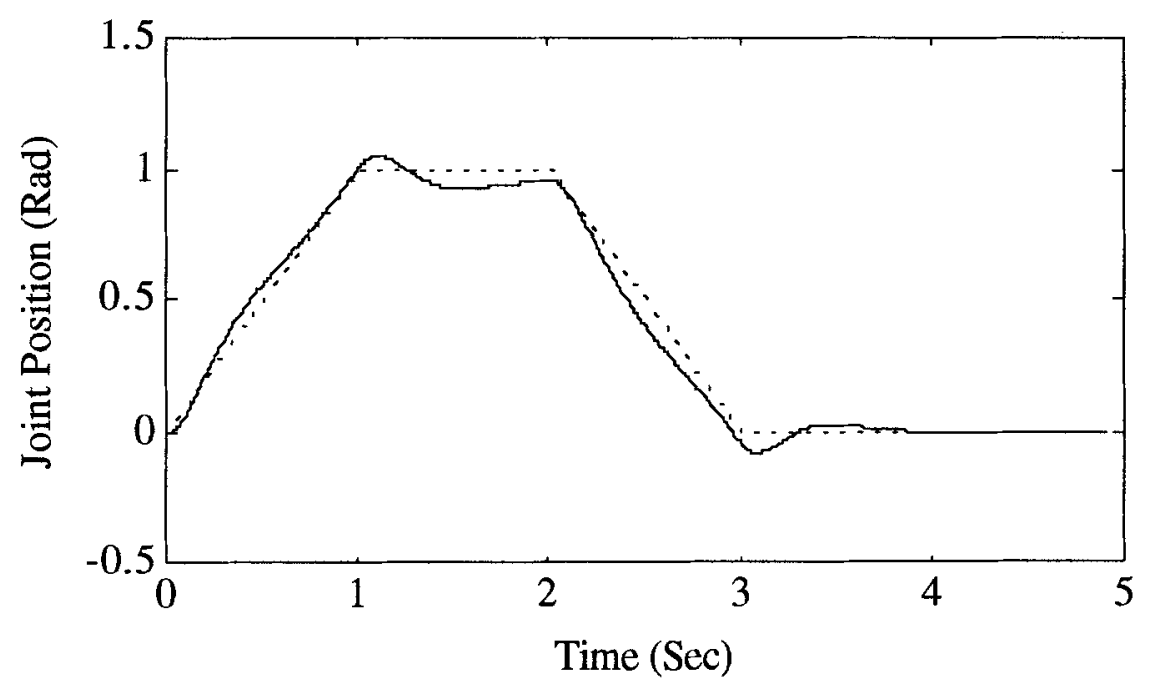

Figure 5. The tracking performance of a versatile sliding control $\left(\zeta_{l}=1\right.$ and $\left.z_{0}=-1\right)$ (a dotted line for desired trajectory).

further decreased $z_{0}$ (Figure 5), the more active $s$ dynamics due to thicker boundary layer cause greater tracking error. The tracking performance with $z_{0}=1$ (Figure 4) is therefore better than those with $z_{0}=100$ and $z_{0}=-1$ (Figures 3 and 5). Additional tuning can be performed through an adjustment of $\zeta_{l}$ in order to improve the tracking performance. The greater the value of $\zeta_{l}$, the thinner the boundary layer and the less overshoot for the $s$ response. Figures 6(a)-6(b) present an improved tracking performance and the associated control input with $\zeta_{l}=10$ and $z_{0}=1$.

For comparison the performance of the first-order sliding control was also examined, where the $s$ dynamics is first-order. The bandwidth of the dynamics was assigned 10 $(\mathrm{rad} / \mathrm{sec})$ and the first pole was also assigned -10 . Note that the the bandwidth of the $s$ dynamics of this control was chosen to be greater than that of the second-order sliding control system. Figure 7 presents the tracking performance of the associated continuous sliding control. One way to reduce the tracking error is by implementing sliding control with integral action. The tracking performance is shown in Figures 8. By selecting an additional identical pole, i.e., second pole $-p_{2}=-10$, for the error dynamics in order to have a critically-damped response, the tracking pattern shows an undesirable overshoot. The integral action destabilizes the error response and causes the oscillation. With more damping added to the error dynamics, the overshoot may disappear but results in sluggish motion. The results will be shown later when point-to-point control is mentioned. As the bandwidth of the $s$ dynamics is reduced to $1(\mathrm{rad} / \mathrm{sec})$, high-frequency uncertainties would be better filtered by the low-bandwidth $s$ dynamics (a first-order low-pass filter). Unfortunately, the motion turns out having greater tracking error (Figure 9) and there is not enough capability to fine tune the $s$ dynamics. 


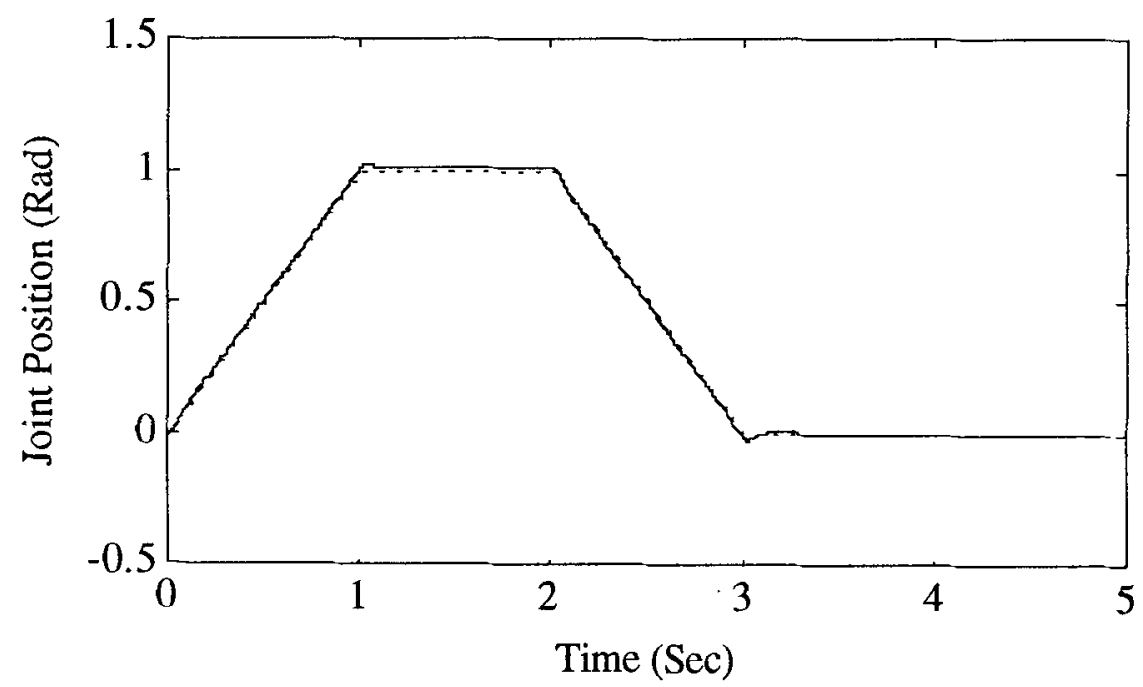

Figure $6 a$. The tracking performance of a versatile sliding control with $\zeta_{t}=10$ and $z_{0}=1$ (a dotted line for desired trajectory).

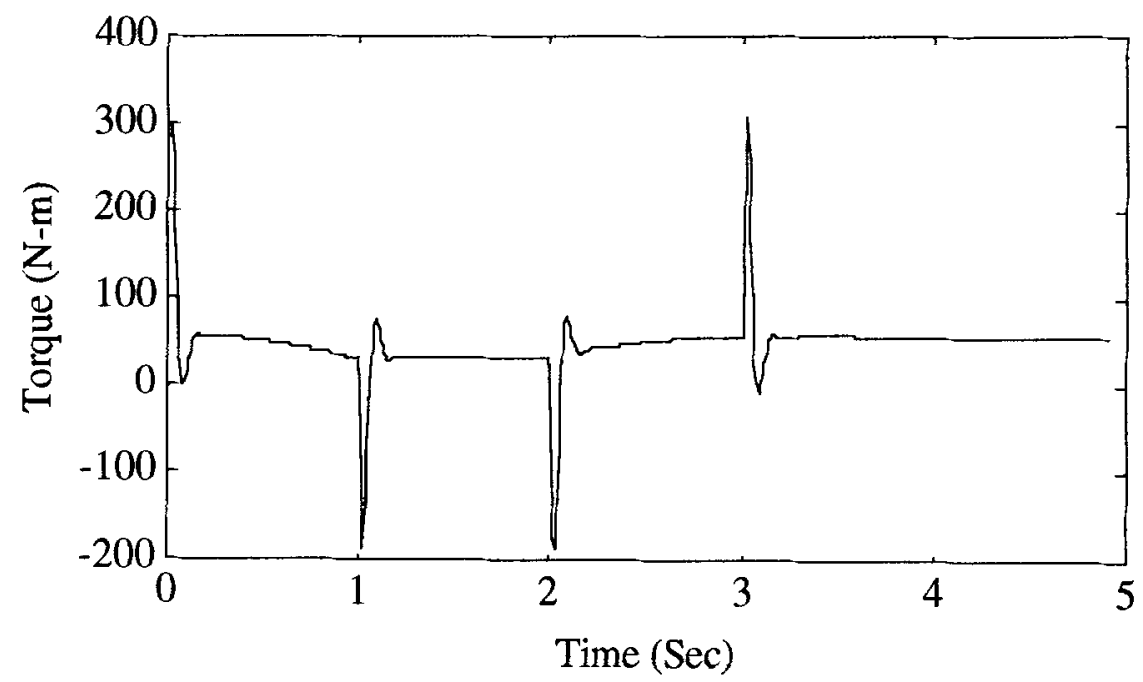

Figure $6 b$. The control input of a versatile sliding control with $\zeta_{l}=10$ and $z_{0}=1$.

When a requirement of no (or minimum) overshoot motion response is imposed, the versatile sliding control has superior capability to keep the speed, which is illustrated by the point-to-point control. Even under the severe test (Maximum payload), Figure 10 shows a good point-to point control performance. Note that some reserved capability, e.g., the control parameters $z_{p}$ in Equation (3.6), can further be used to bring the overshoot down 


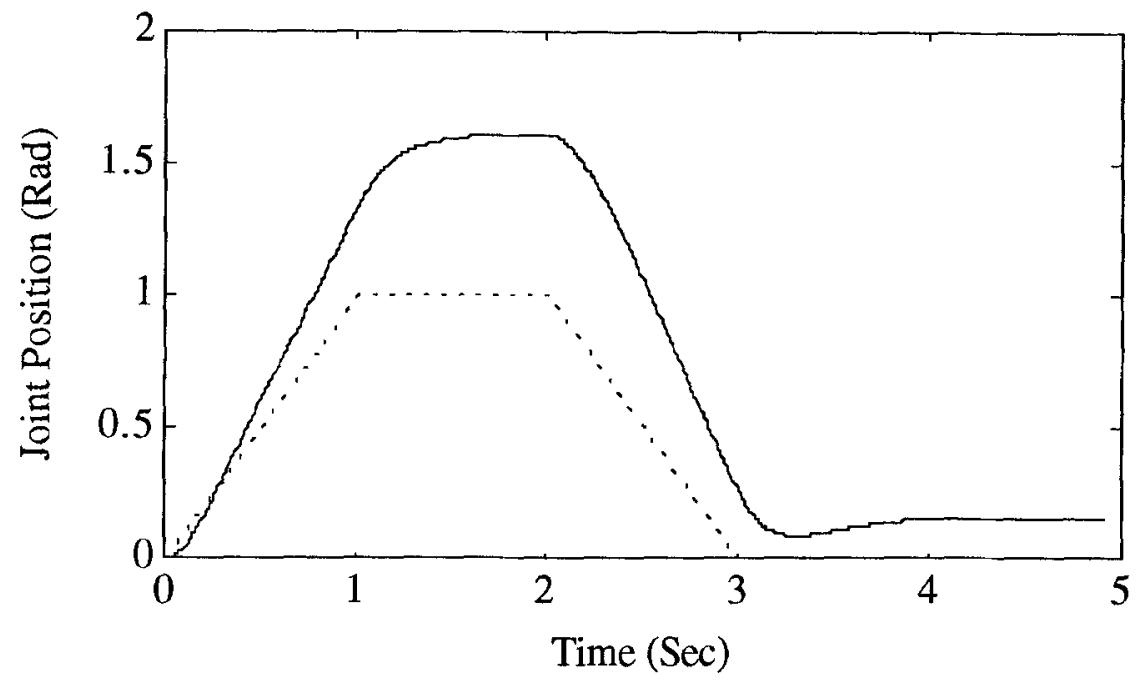

Figure 7. The tracking performance of a continuous sliding control with a first-order sliding condition (a dotted line for desired trajectory).

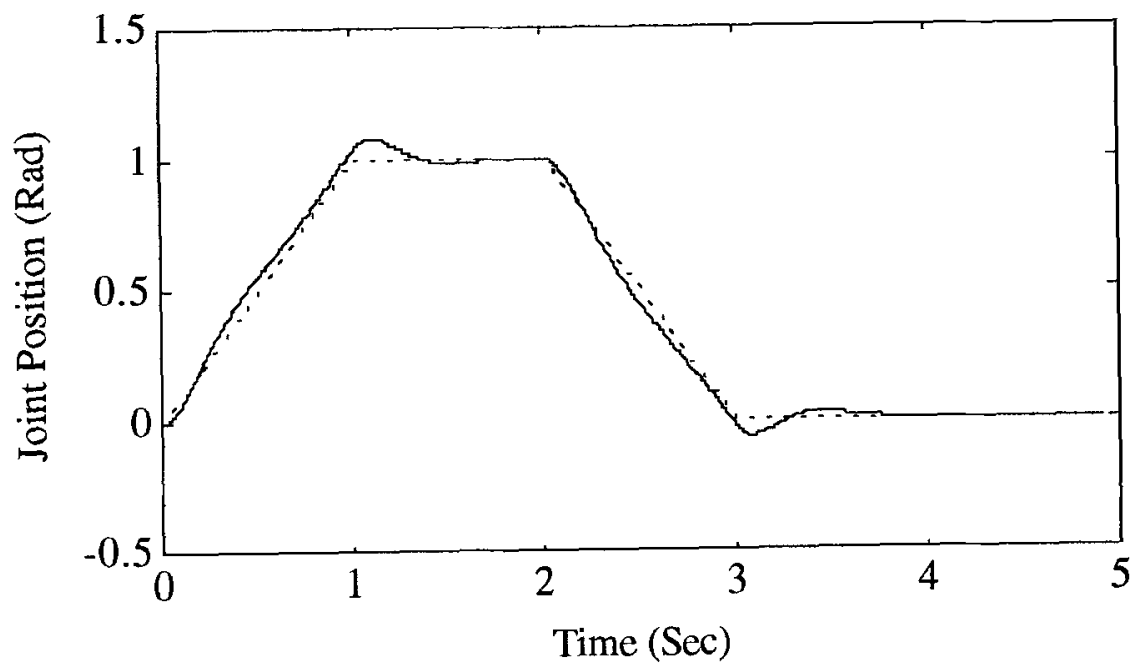

Figure 8 . The tracking performance of a continuous integral sliding control with a first-order sliding condition (a dotted line for desired trajectory).

to zero if it is necessary. To show the contrast, a sliding control with a first-order sliding condition presents a slower step response (Figure 11), i.e., longer settling time, where the damping ratio of the error dynamics has to be increased to 10 or more in order to meet the minimum overshoot requirement. Also, the latter control tends to be more sensitive to the size of the time interval in the numerical integration. With the same size of the time 


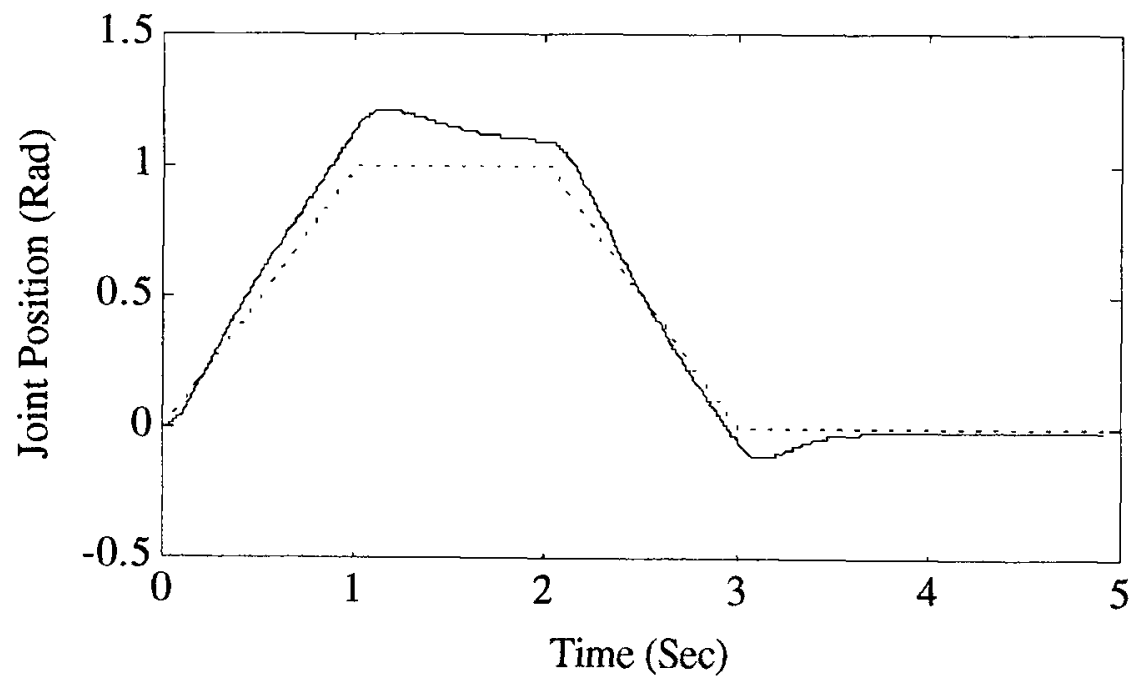

Figure 9. The tracking performance of a continuous integral sliding control with a first-order sliding condition and a low-bandwidth $s$ dynamics (a dotted line for desired trajectory).

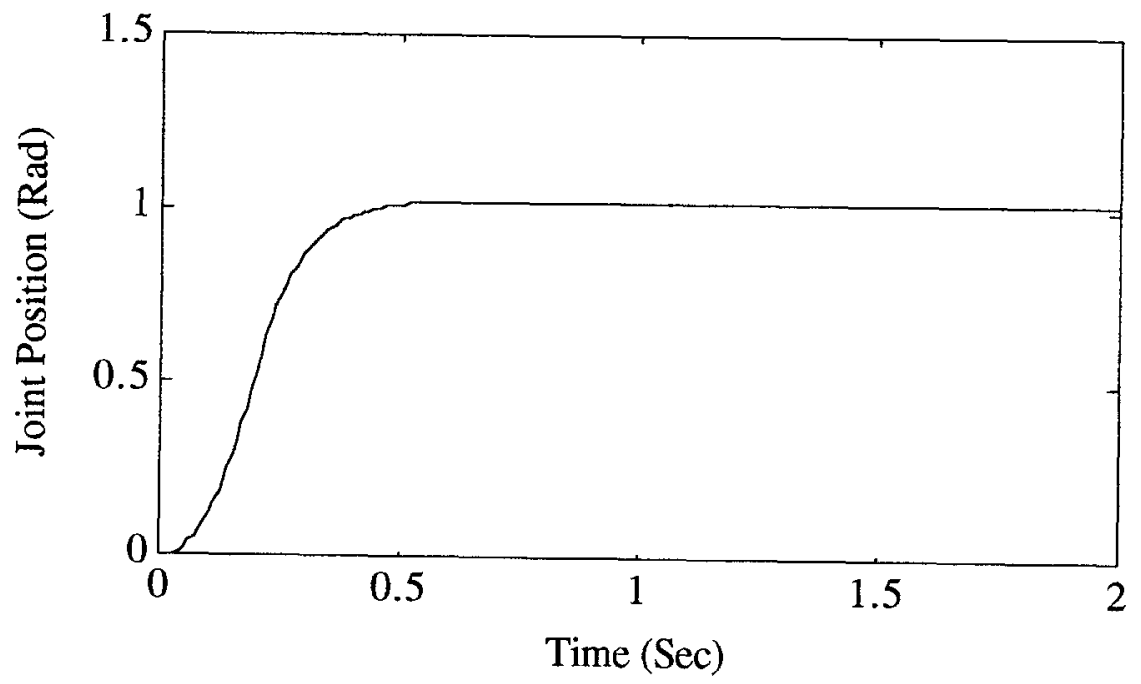

Figure 10. A step response of a versatile sliding control $\left(\zeta_{l}\right.$ and $\left.z_{0}=10\right)$.

interval (i.e., $0.01 \mathrm{sec}$ ), the control input presents an oscillatory response. To eliminate the oscillation, the time interval needs to be chosen smaller. In other words, the versatile sliding control may be more robust (more insensitive to the digitization) in numerical integration. This implies that the versatile sliding control may allow for slower sampling rate in digital control. 


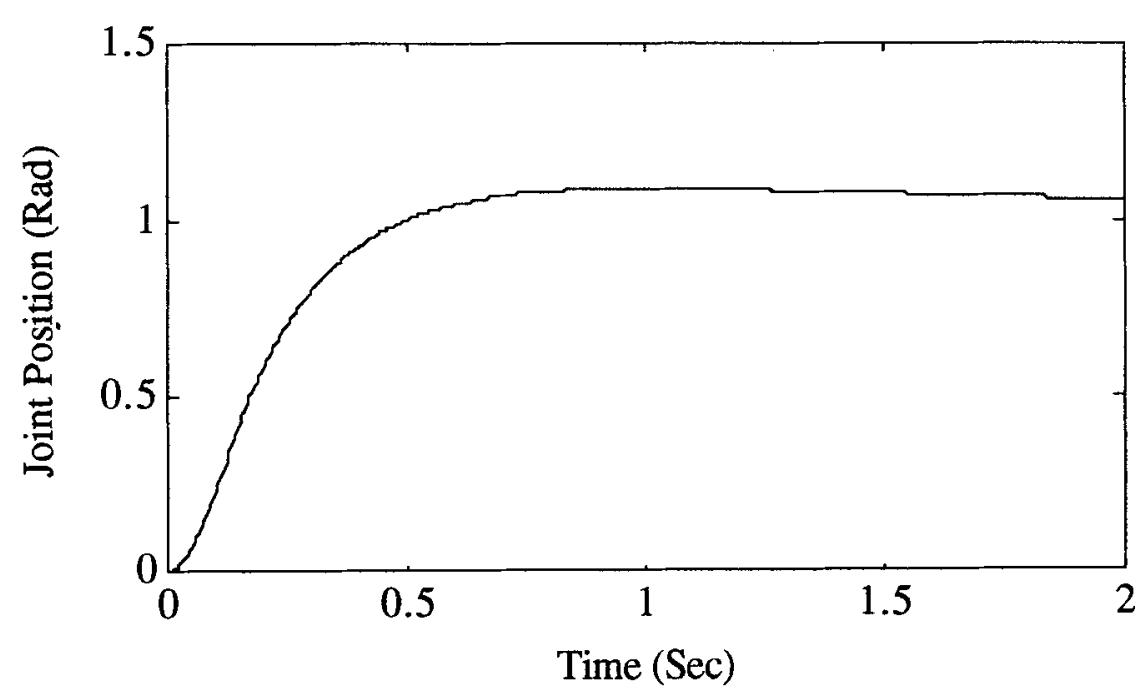

Figure 11. A step response of a continuous sliding control with a first-order sliding condition (damping ratio of the error dynamics is set 10 to meet minimum overshoot requirement).

It is worthwhile to mention that all results presented above were obtained based on only one measurement, the joint position. The sliding control is robust enough to be implemented with a lesser cost in hardware installation. The joint speed was generated from a simple estimator, a numerical differentiation, that is noisier than the direct measurement. It is our intention to put the control in a severe test where only this primitive estimator was integrated with the system. It was observed that the response with two measurements, position and speed, is only slightly smoother.

\section{Conclusion}

A versatile sliding control algorithm has provided a systematic and simple way to design a control system containing both robustness and performance. The structure of the sliding control system is composed of $s$ dynamics and error dynamics, where the $s$ dynamics is designed to be a low-pass filter and the error dynamics is designed to be a high-pass (or band-pass) filter. The bandwidth of the $s$ dynamics is selected to be low so that the error dynamics were not significantly excited. On the other hand, high-frequency uncertainties (unmodeled dynamics, error dynamics, and disturbances) are filtered by the low-pass filter. Low-frequency uncertainties are then filtered by the high-pass filter. The $s$ dynamics can be fine tuned by the lower bound of damping ratio and the placed zero. Therefore, the trade-off between tracking accuracy and robustness to the uncertainties no longer exists. However, the high-frequency induced by the sampling process (in digital control) might enter into the error dynamics and care must be taken to choose a proper sampling rate for a digital control system. In addition, it was found that the control is very sensitive to the saturation of the actuator; therefore, it is suggested that any control input saturation be avoided. 


\section{Acknowledgment}

This research was conducted with direct funds administered by the Naval Postgraduate School and sponsored by the Naval Research Laboratory.

\section{References}

1. Ambrosino, G. et al., "Variable Structure Model Reference Adaptive Control Systems," Int. Journal of Control, vol. 39, pp. 1339-1349, 1984.

2. Chang, L. W., "A MIMO Sliding Control with a Second-Order Sliding Condition," 90-WA/DSC-5, ASME Winter Annual Meeting, Dallas, TX, 1990.

3. DeCarlo, R. A., et al., "Variable Structure Control of Nonlinear Multivariable Systems: A Tutorial," in Proceedings of the IEEE, vol. 76, pp. 212-232, 1988.

4. Dorling, C. M. and Zinober, A. S. I., "Two Approaches to Hyperplane Design in Multivariable Variable Structure Control Systems," Int. Journal of Control, vol. 44, pp. 65-82, 1986.

5. Fernandez, B., and Hedrick, J. K., "Control of Multivariable Non-Linear Systems by the Sliding Mode Method," Int. Journal of Control, vol. 46, pp. 1019-1040, 1987.

6. Itkis, U., Control Systems of Variable Structure, Halsted Press, 1976.

7. Slotine, J. J., and Sastry, S. S., "Tracking Control of Non-Linear Systems Using Sliding Surfaces, with Application to Robot Manipulators," Int. Journal of Control, vol. 38, pp. 465-492, 1983.

8. Slotine, J. J., "Sliding Controller Design for Non-Linear Systems," Int. Journal of Control, vol. 40, pp. 421434, 1984.

9. Slotine, J. J., "The Robust Control of Robot Manipulators," The International Journal of Robotics Research, vol. 4, pp. 49-64, 1985.

10. Utkin, V. I."'Survey Paper-Variable Structure Systems with Sliding Modes," IEEE Trans. Automatic Control, vol. AC-22, pp. 212-222, 1977.

11. Utkin, V. I., Sliding Modes and Their Application in Variable Structure Systems, English Translation, Mir Publishers, Moscow, 1978.

12. Yoerger, D., and Slotine, J. J., "Robust Trajectory Control of Underwater Vehicles," IEEE Journal of Oceanic Engineering, vol. OE-10, pp. 462-470, 1985. 